\title{
Modelación y gestión de una propuesta pedagógica (MAAE: medios audiovisuales aplicados a la educación) para el rescate y fortalecimiento de valores sociales en estudiantes de grado cuarto de la institución educativa Santa Cruz de Lorica- Córdoba, Colombia
}

\author{
José Antonio Correa Padilla, Universidad de Córdoba, Colombia
}

\begin{abstract}
Resumen: La presente investigación busca rescatar, fortalecer y formar en valores humanos, estimula a un cambio axiomático que es favorable para todos. Y es el de solventar la clara necesidad de establecer un ambiente y un vínculo sano entre los estudiantes y el profesor, estudiantes entre sí, al igual que estudiantes y padres de familia, los cuales junto a los maestros y la sociedad deben educar bien a los niños, con la mejor intencionalidad en la actividad normal de la relación humana afectiva, de alli que en la ejecución del presente proyecto se nos invite a replantear nuestro quehacer Docente, asumiendo un papel más crítico y real frente a la formación de personas más allá de los contenidos. La manera más segura de mejorar es inculcando valores humanos, tan reales como indispensable, fortaleciendo los valores de los niños de grado cuarto de Básica Primaria de la Institución Educativa Santa Cruz de Lorica, en este caso mediante la implementación de una propuesta pedagógica denominada MAAE (Medios Audiovisuales Aplicados a la Educación) que se exterioriza como una mediación entre recursos y estrategias significativa incluyendo la gestión, uso y creación de DPS: Documentales Sociales Participativos sobre temáticas arraigadas a necesidades y problemáticas que se presentaban en el contexto, mediante historias libres y expresiones espontáneas de los investigados, así como el uso de fotografias, videos etc., donde se logró determinar la incidencia que tuvo la ejecución de la estrategia propuesta y a partir de ella el generar gran motivación, reflexión crítica, cambios de pensamiento, comportamiento y actitud, mejorando su ambiente escolar, familiar y social y a su vez contribuyendo al uso y apropiación de los materiales audiovisuales aplicados a la educación.
\end{abstract}

Palabras clave: tecnologías de la información y la comunicación, valores, formación en valores, lúdicas, medios audiovisuales, DPS: Documentales Sociales Participativos

\begin{abstract}
This research seeks to rescue, strengthen and form human values, encourages an axiomatic change that is favorable for all. And is to overcome the clear need to establish an environment and a healthy bond between the students and the teacher, students among themselves, as students and parents, which together with teachers and society must educate well to children, with the best intentions in the normal activity of human affective relations, hence the implementation of this project will invite us to rethink our teaching work, taking a more critical and actual role against the formation of people beyond contents. The surest way to improve is to inculcate human values, as real as essential to strengthening the values of fourth grade children Basic Primary Education Institution Santa Cruz de Lorica, in this case through the implementation of a teaching proposal called MAAE (Applied to Audiovisual Media Education) which holds as a significant mediator between resources and strategies including management, use and creation of DPS: Participatory Social documentaries on rooted issues to needs and problems presented in context, with free stories and expressions spontaneous investigated, as well as the use of photographs, videos etc., where it was determined that the incidence was implementing the proposed strategy and from it to generate great motivation, critical thinking, changes in thinking, behavior and attitude, improving their school, family and social environment and in turn contributing to the use and ownership of audiovisual materials applied to education.
\end{abstract}

Keywords: Information Technology and Communication, Values, Values Education, Entertainment, Audiovisual Media, DPS: Participatory Social Documentary

Revista Internacional de Tecnologías en la Educación

Volumen 2, Número 2, 2015, <http://sobrelaeducacion.com>, ISSN 2386-8384

(C) Global Knowledge Academics. José Antonio Correa Padilla.

Todos los derechos reservados. Permisos: soporte@gkacademics.com 


\section{Introducción}

$\mathrm{L}$

as metamorfosis tecnológica y social e la que estamos sumergidos en pleno siglo XXI afecta sin duda a la educación de múltiples formas; este desarrollo tecnológico impresiona a todas las áreas de la vida y a todos los miembros de la comunidad, generalmente el colegio se había centrado fundamentalmente en transmitir una serie de contenidos conceptuales, pero actualmente se hace necesario enseñar también una serie de valores personales, escolares y comunitarios que permitan a nuestros estudiantes desenvolverse en una sociedad en constante cambio y de esta manera entender cómo actuar sobre determinados comportamientos que se consideran valores básicos para la convivencia, necesarios tanto en el aula como en la sociedad y que contribuyan a fomentar el respeto, el diálogo y la sensibilización ante los problemas que a diario se presentan en un determinado contexto.

Es necesario entonces que las instituciones educativas actualicen su metodología para afrontar este gran reto como parte de la socialización cultural de los niños y niñas cada vez en mayor medi$\mathrm{da}$, aprovechando las distintas tecnologías de la información que utilizan prácticamente desde que nacen (televisión, vídeo, videojuegos, móvil, Internet).

Debido a que los actuales ciudadanos menores de diez años son, en este sentido, la primera generación de la llamada sociedad de la información.

En consecuencia, esta nueva generación cada vez aprende más cosas fuera de la escuela a través del uso de las distintas tecnologías audiovisuales e informáticas, es por eso que la presente investigación utiliza como herramienta los medios audiovisuales ya que es una forma muy llamativa para los estudiantes, que les proporciona aportes útiles y prácticos que inciden en la modificación de comportamientos en la población escolar, fortaleciendo valores, permitiendo la reflexión crítica y motivando de manera controlada la libre expresión de sentimientos que los lleve a una convivencia sana.

\section{Planteamiento del problema}

\subsection{Descripción del problema}

Los estudiantes de la institución educativa santa cruz de lorica ostentan un bajo índice de formación en valores sociales imprescindibles en la formación integral de los seres humanos, esto evidenciado en un vocabulario irrisorio y la detección de un nivel de antivalores bastante alto entre los estudiantes de la población objeto de estudio, mostrada en las sesiones de descanso e incluso en el aula de clase, como respuesta a esto se requiere de una motivación que lleve a un cambio de actitud. De muchos estudiantes.

Mediante una observación contextual, se pudo analizar que esa problemática estaba ligado a la escasa educación en valores humanos impartida en sus hogares y en las comunidades donde viven, que en la mayoría son invasiones o lugares marginados de la ciudad.

Es allí donde entra el papel del docente como formador integral del ser humano y donde se hace necesaria la búsqueda de estrategia pertinente para solventar esta problemática.

Esta investigación promueve, la exploración de la verdad, la armonía, la responsabilidad, el respeto, la solidaridad, amistad, comprensión mutua, tolerancia, honradez, y muchos valores que por medio de medios audiovisuales se convierten en herramientas llamativas para la formación integral de la comunidad y el mejoramiento de ambientes armónicos de convivencia, además que se incrementó el interés por el uso de materiales audiovisuales en las clases por parte de docentes, y la participación activa en la creación de documentales sociales participativos donde se pude exponer realidades a las que están expuestos estos estudiantes.

\subsection{Formulación del problema}

¿De qué manera mediante la implementación la propuesta pedagogía MAAE: Medios Audiovisuales Aplicados a la Educación se pueden fortalecer los valores sociales de la comunidad educativa de los estudiantes de grado cuarto de la institución educativa santa cruz de lorica? 


\section{Justificación}

Dado que el entorno en el cual se desarrolla la vida de muchos estudiantes no es el óptimo, debido a situaciones como: violencia intrafamiliar, poco fortalecimiento de las habilidades para la vida, situaciones de injusticia social, degradación de los valores, irrespeto a los derechos de los niños y niñas, inadecuado manejo de conflictos entre otras; surge la necesidad de brindar en los estudiantes de grado cuarto de primaria en la Institución Educativa santa cruz de lorica un ambiente que contrarreste las falencias anteriores y que permita que nuestros estudiantes crezcan en el enriquecimiento de valores que hagan de ellos y de quienes los rodean personas felices.

El proyecto MAAE involucra en primera instancia a los escolares, quienes son los actores principales de la presente investigación, donde ellos serán artífices e investigadores para encontrar los antivalores que más se presentan en su desempeño y convivencia y en este mismo sentido en sus compañeros y así poder ayudar a plantear y formular estrategias que superen las falencias encontradas en su crecimiento individual y social en el medio en el cual se encuentran inmersos.

Partimos del hecho de que como docentes en formación de la licenciatura en informática y medios audiovisuales se hace necesario apoyarse de los componentes curriculares del pregrado, más exactamente los módulos de medios audiovisuales e implementarlos de manera metodológica e innovadora para solventar problemas que acechan al aula de clase, puesto que sabemos que el uso de medios audiovisuales en la educación da un gran impulso al proceso de enseñanza-aprendizaje en muchos ámbitos.

Sabemos que el uso de recursos iconográficos o sonoros como fotografías, videos, esquemas, figuras, etc. da una nueva tonalidad a este proceso en el sentido de que los estudiantes adquieren un interés mayor por los temas expuestos. Por su parte, el video o los documentales se utilizan como apoyo a la reflexión de temas o desarrollo de habilidades analíticas dentro de los estudiantes, concibiendo al video no como distractor, sino como un soporte didáctico.

Para la ejecución de materiales didácticos audiovisuales es importante tener en mente una serie de discernimientos y características no solo con respecto a los contenidos, sino también con respecto a discreciones y técnicas que admitan una producción de calidad enfocada a la mejora del proceso de enseñanza aprendizaje, en el caso de esta investigación al fomento de valores y la formación del ser integro.

\section{Objetivos}

\subsection{General}

Determinar la incidencia que tiene el uso la propuesta pedagógica MAEE (Medios Audiovisuales Aplicados a la Educación) en el fortalecimiento de valores sociales en los estudiantes grado cuarto de educación básica primaria de la Institución Educativa Santa Cruz de Lorica.

\subsection{Específicos}

- Implementar herramientas audiovisuales al aula de clase mediante foros y debates sobre la innovación en proyectos de pedagogía audiovisual.

- Conocer el impacto que poseen los medios audiovisuales como recursos metodológicos para el docente.

- Diseñar historias basadas en temas sociales y experiencias personales para la realización de DPS: Documentales Sociales Participativos para analizar la temática de estudio.

- Realizar con los estudiantes actividades que permitan observar cambios de actitud frente a situaciones determinadas.

- Conformar el Semillero de investigación MAAE para promover la cultura investigativa en la Institución Educativa Santa Cruz de Lorica. 


\section{Marco referencial}

\subsection{Antecedentes}

Carretero (2012), en el desarrollo de la investigación "La preservación de los Derechos humanos y los Medios Audiovisuales en el IE Villa de Vícar", nos relata su aventura pedagógica a través de la web 2.0 y los recursos audiovisuales. En gran medida el proyecto cumplió con todos los objetivos planteados por el investigador y tuvo como como base el inculcar la educación en valores y el respeto y preservación de los Derechos Humanos en el aula, la investigación con diseño IAP logró evidenciar el objetivo principal cumplido mediante fases que era buscar y crear recursos que valieran de una manera práctica en las clases y con ellos mejorar tanto el rendimiento académico de los alumnos como sus relaciones interpersonales, ya que gran parte del proyecto desarrollado tiene como base el inculcar la educación en valores, el respeto y preservar de los Derechos Humanos.

Por otro lado la corporación AIKEN en 2014 con su proyecto Cine Arte (antes Cine Para Todos) lleva a disfrutar del séptimo arte a miles de niños y niñas de sectores vulnerables y excluidos de Montería y Córdoba, en una estrategia por masificar el acceso a nuevas tecnologías y entretenimiento a la población más necesitada de la región.

El programa que surgió en 2010 y ha tenido na gran acogida en el departamento se realiza una vez al año gracias al apoyo de Cine Colombia. Desde su creación, Cine Arte ha movilizado y acercado a la experiencia del cine a más de 13.000 niños y niñas en condición de vulnerabilidad y/o socialmente excluidos. Este año beneficio a 8 mil niños de las instituciones educativas oficiales de Montería, completando así 21.000 niños entre 2010 y 2014.

Palencia (2008), en el desarrollo del proyecto de investigación "MEDUSA, Modelo para la gestión de tecnologías en la educación", logró permear diferentes proyectos a una o varias gestiones determinadas. En ese sentido se logró vincular y beneficiar a todos los actores de la comunidad educativa: Padres, directivos, estudiantes, docentes y egresados.

Este macro proyecto que atendió a la necesidad de articular las tecnologías de la información y la comunicación en las diferentes gestiones institucionales, a través de la implementación de proyectos que atienden a planteamientos o situaciones problemitas detectadas en la evaluación institucional y sistematizadas en el plan de mejoramiento.

Las experiencias abordadas desde MEDUSA, fueron 5 y cada una estuvo dirigida una población diferente (Egresados, estudiantes, padres, docentes y directivos).

En ese sentido se aplicaron la política de TIC y Medios Audiovisuales en la educación, dinamizando los diferentes ejes que desde la Secretaria de Educación municipal se construyen e implementan con el equipo de GESTORES TIC logrando así resultados efectivos y significativos en el uso y apropiación de las TIC y os medios audiovisuales en el contexto escolar.

Del mismo modo, Palencia (2009) realizó el microproyecto "IM@GÍNATE. Mil maneras de aprender", que estuvo adscrito al macroproyeto MEDUSA, dio respuesta al interrogante planteado en la investigación sobre ¿Qué estrategias apoyadas en la producción audiovisual e hipermedia se pueden desarrollar con los estudiantes para la generación de escenarios significativos y activos para su proceso de aprendizaje?, donde se desarrolló la estrategia mediante una metodología IAP: investigación acción participación, logrando vincular a los docentes, estudiantes, padres, directivos, egresados en los procesos de transformación de la realidad, todo eso desarrollado mediante a siguientes fases: Planeación, Acción, Observación y reflexión.

La UNESCO (2010), en el desarrollo de su plan de acción denominado Teaching and Learning for a Sustainable Future, dentro del módulo numero veintidós Values in Education, nos exhibe unas actividades estratégicas para apoyar procesos educativos fundamentado en valores humanos, todo con la finalidad de mostrar lo importante de los valores en los procesos de formación, ya que estos afectan la forma en que nos relacionamos con otras personas y para todas nuestras actividades en el medio ambiente, y por lo que son una gran influencia en nuestras perspectivas para lograr un futuro sostenible.

El modulo se distribuye en una comprensión cognitiva relacionada con los valores y actitudes que se refieren a la afectivo (o emocional) en las dimensiones del comportamiento humano. 
Los objetivos de este módulo que fue aplicado a instituciones educativas de Paris, Francia, estuvo como objetivos en primera instancia desarrollar una comprensión de las estrategias de educación valores; y como específicos estuvo orientado al análisis de la relación entre los valores y el comportamiento personal y si estos afectan a la consecución de un futuro sostenible; otro objetivo fue el Desarrollar habilidades para el uso de la clarificación de valores y análisis de los valores en la enseñanza.

Guzmán (2005) ejecutó unos lineamientos gerenciales para la implantación de las tecnologías de información y comunicación en los procesos educativos de la Escuela Básica "Francisco Anicato Lugo" del sector II, distrito $\mathrm{N}^{\circ} 1$, Municipio Tucupita, Estado Delta Amacuro, cuyo objetivo de la investigación estuvo orientado a la formación de ciudadanos de bien, mediante el conocimiento de los aportes de la tecnología en la educación básica, a través de guías o estándares que los docentes siguieron en sus clases lo cual dio como resultado a estudiantes más proactivos frente a situaciones de competencias ciudadanas.

Incorporación de docentes al uso de la informática y la telemática en la dinámica escolar. Experiencia de investigación acción en el colegio Fe y Alegría María Rosa Molas de Catia. (Cárdenas, 2005).

Bustos y Ramos (2009) diseñan propuestas de estrategias en diversos planteles donde el docente orienta a sus alumnos y los motiva en su vocación; reflejando esta investigación la importancia que tiene la acción docente en el logro de objetivos que le permite al estudiante sentirse cómodo, seguro, percibir el incentivo, recibir afecto, solidaridad, respeto y confianza.

\section{Diseño metodológico}

\subsection{Tipo de investigación}

Estudio de corte cualitativo bajo una perspectiva descriptiva basado en una evaluación de experiencias por medio de técnicas de observación y registro de las dificultades más frecuentes en la presencia de antivalores marcados en la población de estudio.

Este tipo de investigación de carácter descriptivo puesto que pretende mostrar la eficacia de implementar una propuesta metodológica que favorezca el fortalecimiento y rescate de valores sociales en los estudiantes de grado cuarto d en la Institución Educativa Santa Cruz de Lorica.

Para el desarrollo del proyecto se considera un diseño IAP investigación Acción Participación que Implica un proceso de aprendizaje, ya que el objetivo es el poder estar con el otro, el formar comunidad de aprendizaje mediado por Medios Audiovisuales y el concientizarse de la necesidad de vivir armónicamente y obtener un mejor rendimiento académico.

La Investigación es Descriptiva porque se presenta como un ciclo continuo, ya que el problema a investigar es la "Incidencia de los medios audiovisuales aplicados a la educación como herramienta el rescate y fortalecimiento de valores sociales de los estudiantes de la sección primaria de la Institución Educativa Santa Cruz de Lorica del municipio de Lorica, Córdoba"; tiene en cuenta que el estudiante como ser humano tiende a presentar antivalores en sus comportamientos y que es labor del docente ser un sembrador constante de valores que los formen integralmente como mejores seres humanos.

\subsection{Participantes}

La población correspondió a los estudiantes de los grados cuartos de primaria de la Institución Educativa Santa Cruz de Lorica del municipio de Lorica en el departamento de Córdoba.

\subsubsection{Características de los participantes}

Estudiantes de grado 4 con edades que oscilan entre 8-11 años, rendimientos académicos regulares en un $70 \%$, con un bajo nivel de disciplina por sus comportamientos de rebeldía, provenientes de familias con estratos socioeconómicos bajos, y un alto nivel de desigualdades sociales.

El grupo de trabajo estuvo conformado por 16 niños y 9 niñas, con diversos estilos de aprendizaje, con una actitud de irrespeto para con los compañeros y profesores, que en gran medida es 
consecuencia de los problemas intrafamiliares por los que atraviesan en sus hogares, y la escasa educación en valores que sus padres le proporcionan.

\subsubsection{Muestra}

La muestra correspondió a 25 estudiantes del grado 4 de la Institución Educativa; por ser aquel del que se han recibido mayores comentarios de demostración de antivalores según lo informado por los docentes y lo confirmado en una observación contextual.

\subsubsection{Línea de investigación}

En el documento maestro de la Licenciatura en informática y medios audiovisuales se nos manifiesta que as investigaciones de la facultad de Educación y Ciencias Humanas de la cual hace parte el programa tributan a la Línea de investigación Institucional: EDUCACIÓN, CULTURA Y SOCIEDAD como se estable en el Estatuto de investigación y extensión, Universidad de Córdoba.

Partiendo de allí y de la estructura de la investigación se afirma que la presente investigación encaja en la línea de investigación de la licenciatura "Estudio del Impacto de las tecnologías de la información y comunicación en educación", que tiene por objetivo Evaluar la Incidencia de la Tecnología en la Formación integral del Hombre, desde una perspectiva interdisciplinaria que permita la valoración de enfoques y posturas epistemológicas, sociológicas y/o cognitivas como de orden gerencial en la educación con el apoyo de soportes tecnológicos.

\section{Análisis de datos y resultados obtenidos}

La recolección de datos se hizo a través de encuestas como instrumento válido, conformado por preguntar abiertas que respondieran a la necesidad de incorporar la propuesta dentro de la investigación.

Una vez aplicado el instrumento de recolección de información mediante una fase a la que se le denomino como observación contextual a los estudiantes de cuarto de primaria de la Institución Educativa Santa Cruz de Lorica y analizados los datos, la información obtenida fue:

\section{Interrogantes}

1. ¿Sabe usted identificar lo que son valores?

Tabla 1: ¿Sabe usted qué son valores?

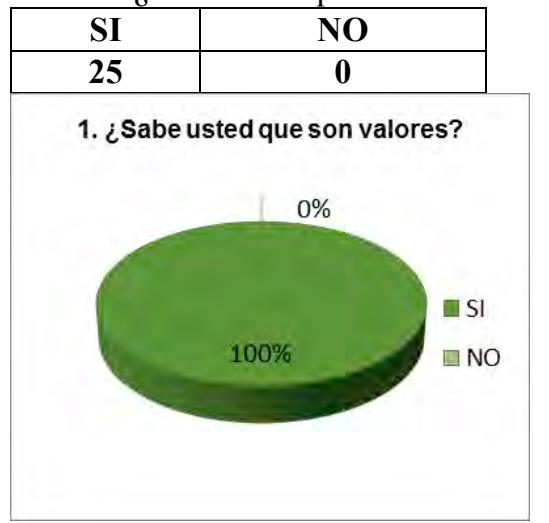

Fuente: Elaboración propia, 2015.

El total de los encuestados respondió afirmativamente a esa pregunta, lo cual corresponde al $100 \%$ de la población. 
2. ¿Considera importante la práctica de valores en su familia?

Tabla 2: ¿considera importante la práctica de valores en su familia?

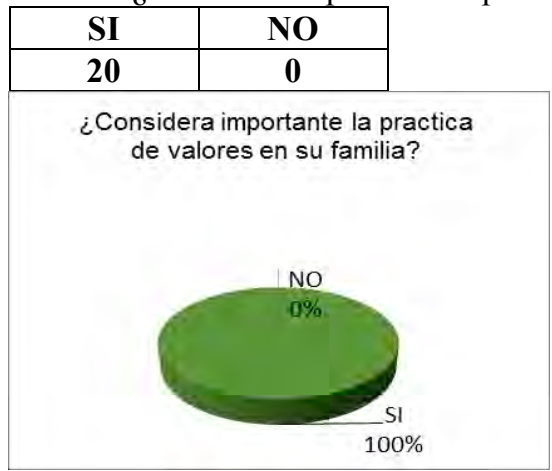

Fuente: Elaboración propia, 2015.

El total de los encuestados respondió que si considera importante la práctica de valores en la familia. Esto corresponde al 100\% de los estudiantes encuestados.

3. Del siguiente listado marque con una " $x$ " los dos valores que considera más importantes

Tabla 3: Del siguiente listado marque con una " $\mathrm{x}$ " los dos valores que considera más importantes

\begin{tabular}{|l|c|}
\hline \multicolumn{1}{|c|}{ opción } & Número de veces seleccionado \\
\hline Respeto & 17 \\
\hline Tolerancia & 10 \\
\hline Amor & 8 \\
\hline Solidaridad & 4 \\
\hline Responsabilidad & 3 \\
\hline Honradez & 0 \\
\hline Comunicación & 0 \\
\hline
\end{tabular}

3. Del siguiente listado marque con una $x$ los dos valores que considera mas importantes

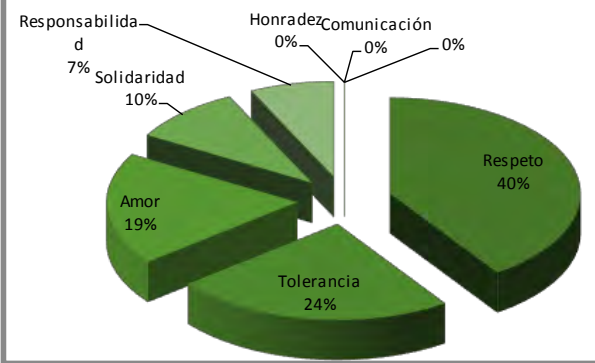

Fuente: Elaboración propia, 2015.

El $40 \%$ de los encuestados que corresponde a 17 estudiantes considera que el respeto es el valor más importante.

El segundo lugar corresponde a la tolerancia equivalente al 24\%, respondido por 10 estudiantes.

En tercer lugar 8 estudiantes escogieron el valor del amor, lo anterior equivale al 19\%.

El cuarto lugar corresponde a la solidaridad equivalente al 10\%, escogido por 4 estudiantes.

En quinto lugar lo ocupa la responsabilidad con 7\%, escogido por 3 de los encuestados. 
Los valores de honradez y comunicación no fueron seleccionados por los encuestados.

4. marque con una " $\mathrm{x}$ " el valor que más lo identifica en el colegio

Tabla 4: Marque con una " $x$ " el valor que más identifica en el colegio

\begin{tabular}{|l|c|}
\hline Opciones & Número de veces seleccionado \\
\hline Respeto & 14 \\
\hline Solidaridad & 5 \\
\hline Tolerancia & 4 \\
\hline
\end{tabular}

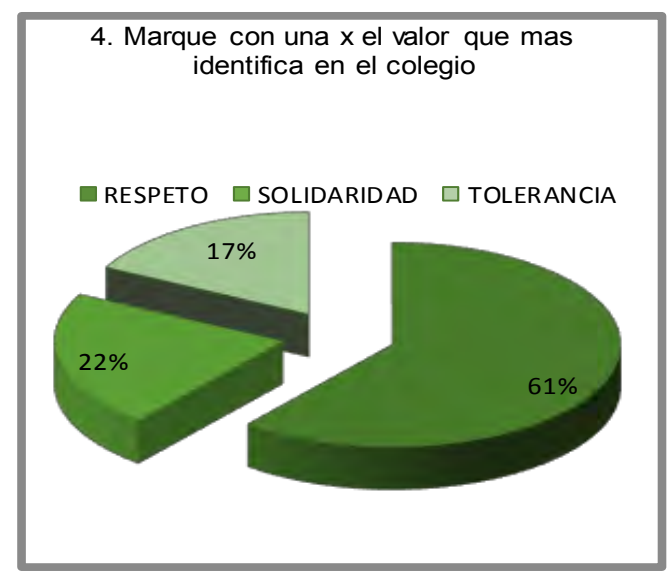

Fuente: Elaboración propia, 2015.

14 estudiantes encuestados que corresponden al 61\% consideran que el respeto el valor que más identifican en el colegio; en segundo lugar se encuentra la solidaridad con $22 \%$, respondido por 5 encuestados y en tercer lugar la tolerancia con un porcentaje de $17 \%$ respondido por 4 encuestados.

5. ¿Sabe usted que es un antivalor?

Tabla 5: ¿Sabe usted qué es un antivalor?

\begin{tabular}{|c|c|}
\hline SI & NO \\
\hline $\mathbf{1 9}$ & 1 \\
\hline
\end{tabular}

\section{Sabe usted que es un antivalor?}

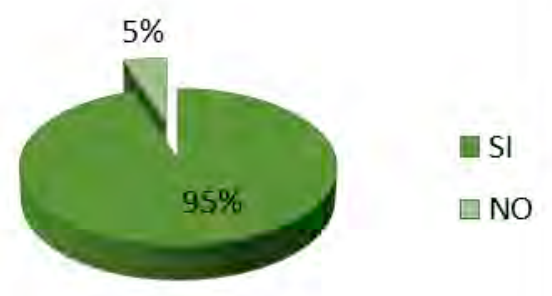

Fuente: Elaboración propia, 2015. 
19 estudiantes encuestados respondieron afirmativamente a esta pregunta, lo que constituye el 95\% y 1 estudiante respondió negativamente, lo que corresponde al 5\%

6. Marque con una " $x$ " el antivalor que más identifica en el colegio

Tabla 6: marque con una " $\mathrm{x}$ " el antivalor que más identifica en el colegio

\begin{tabular}{|l|c|}
\hline \multicolumn{1}{|c|}{ Opción } & número de veces seleccionado \\
\hline Intolerancia & 17 \\
\hline Irrespeto & 15 \\
\hline Deshonestidad & 2 \\
\hline Irresponsabilidad & 1 \\
\hline
\end{tabular}

6. marque con una $x$ el antivalor que mas identifica en el colegio

- INTOLERANCIA $\square$ IRRESPETO

$\square$ DESHONESTIDAD $\square$ IRRESPONSABILIDAD

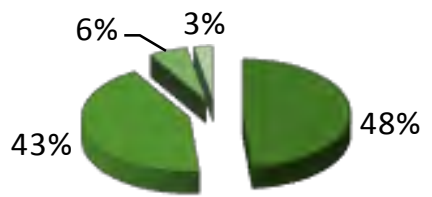

Fuente: Autor, 2015.

La intolerancia ocupa el primer lugar como el antivalor que los encuestados más identifican en el colegio con un porcentaje del $48 \%$ respondido por 17 estudiantes.

15 estudiantes encuestados consideran que el irrespeto ocupa el segundo lugar, esto equivale al $43 \%$

El tercer antivalor de acuerdo a lo respondido por 2 estudiantes es la deshonestidad que corresponde al $6 \%$

La irresponsabilidad corresponde al cuarto lugar, de acuerdo a lo respondido por 1 encuestado, lo que corresponde al $3 \%$.

Tabla 7: ¿La institución educativa brinda espacio para el fortalecimiento de valores?

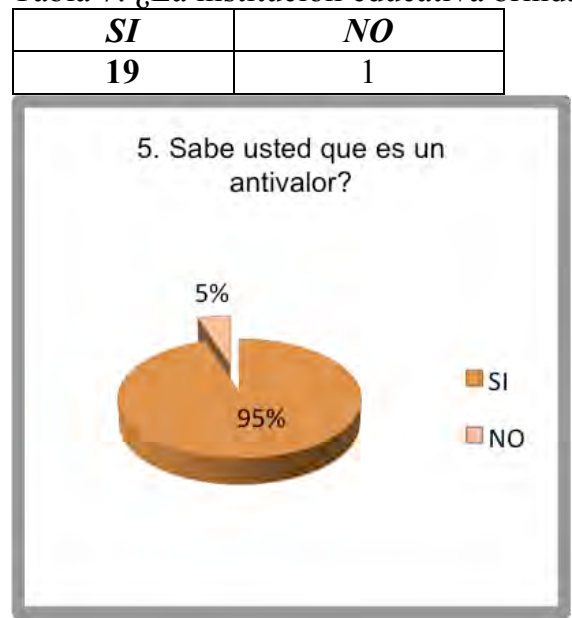

Fuente: Autor, 2015. 
19 encuestados respondieron afirmativamente, lo que corresponde al $\quad 95 \%$ y 1 encuestado respondió negativamente, lo que corresponde al $5 \%$

7. ¿De qué manera cree que se puede motivar para la construcción y fortalecimiento de valores?

Tabla 8. ¿De qué manera cree que se puede motivar para la construcción y fortalecimiento de valores?

\begin{tabular}{|l|c|}
\hline \multicolumn{1}{|c|}{ Opción } & Número de veces seleccionado \\
\hline Videos documentales & 17 \\
\hline EI ejemplo & 6 \\
\hline Imágenes & 3 \\
\hline Conferencias & 2 \\
\hline
\end{tabular}

8. ¿De que manera cree que se puede motivar

para la construccion y fortalecimiento de

valores?

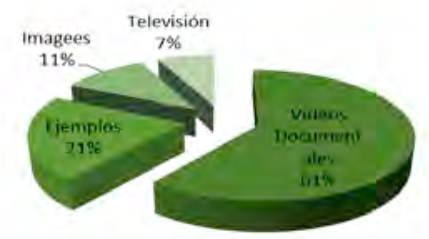

Fuente: Autor, 2015.

17 de los encuestados manifiesta que se puede motivar para la construcción y fortalecimiento de valores a través de videos documentales, esto constituye el $61 \%$.

El ejemplo ocupa el segundo lugar con un porcentaje del $21 \%$ respondido por 6 de los encuestados.

Las imágenes ocupan el tercer lugar con un porcentaje del 11\% y 3 de los encuestados.

Las conferencias ocupan el cuarto lugar con un porcentaje del $7 \%$ respondido por 2 de los encuestados.

8. ¿Considera usted que los medios de comunicación contribuyen en el rescate y fortalecimiento de valores?

Tabla 9: ¿Considera usted que los medios de comunicación contribuyen en el rescate y fortalecimiento de valores?

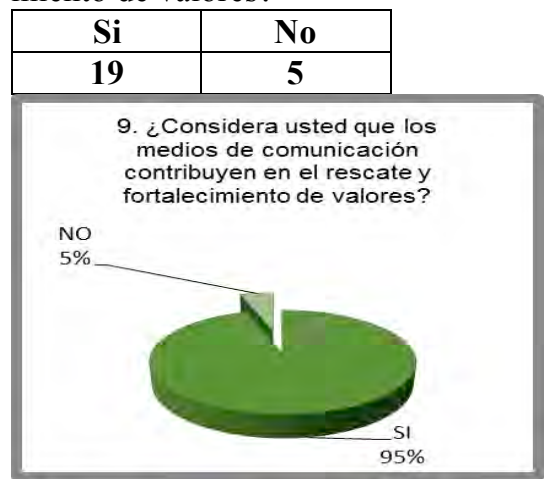

Fuente: Autor, 2015.

19 encuestados respondieron afirmativamente, lo que corresponde al 95\% y 5 encuestado respondió negativamente, lo que corresponde al 5\% 


\section{Resultados}

Los estudiantes de los grados cuartos de la Institución Educativa presentaban una serie de comportamientos inapropiados al igual que el incumplimiento del manual de convivencia lo cual denotaba antivalores presentes en su cotidianidad.

Una vez aplicada la estrategia propuesta pedagogía MAAE se comprobó lo planteado en la hipótesis: "Los medios audiovisuales si motivaron a los estudiantes del grado cuarto de la Institución Educativa Santa cruz de lorica Márquez a modificar sus antivalores y a reflejarlo en sus nuevos comportamientos, fortaleciendo los valores humanos Lo anterior lo corrobora el informe presentado por la Psicóloga de la institución.

Cabe resaltar que los Medios Audiovisuales como herramientas de innovación ofrece una ventana de posibilidades para que el docente se apoye en sus clases, estos sirven para ser implementados dentro de la estructura metodológica de un docente.

Un punto aparte es que se desarrollaron planes de clase enfocados a la implementación de MAAE en diferentes contextos pero de una manera menos rigurosa, involucrando a los estudiantes de práctica profesional I de la licenciatura en informática y medos audiovisuales los cuales ostentan fallas en la metodologías implementadas en su proceso docente y estos evidenciaron una mejoría en su práctica profesional y una valoraciones más altas dentro de la evaluación metodológica por parte del docente asesor de la práctica profesional.

\section{Valoración de cambios}

Ante el notable éxito de la propuesta pedagógica MAAE se procedió a realizar una valoración de cambios para analizar qué perspectivas y recomendaciones tenían los implicados en todo el proyecto, para lo cual se realizó una encuesta de satisfacción que evidenciara los motivos que hacen imprescindible seguir realizando acciones encaminadas al trabajo de metodología por proyectos en el aula de clase y la incorporación de TIC y recursos audiovisuales al mismo.

Cabe resaltar que dentro de esa valoración de cambios se debe reflexionar sobre el ejercicio docente y el magnífico trabajo que estos hacemos, El docente dentro de su ejercicio debe precisar propuestas pedagógicas que fomenten el desarrollo valores humanos para que el estudiante tome conciencia de las propias capacidades y a partir de allí esgrima sus habilidades para proyectar, desarrollar y evaluar el propio aprendizaje, donde se considere motivado y posea confianza en sí mismo necesaria para manejar el aprendizaje de forma autónoma y eficaz en los distintos contextos. 
Tabla 10: ¿Qué calificación le das a los procesos desarrollados en el proyecto MAAE?

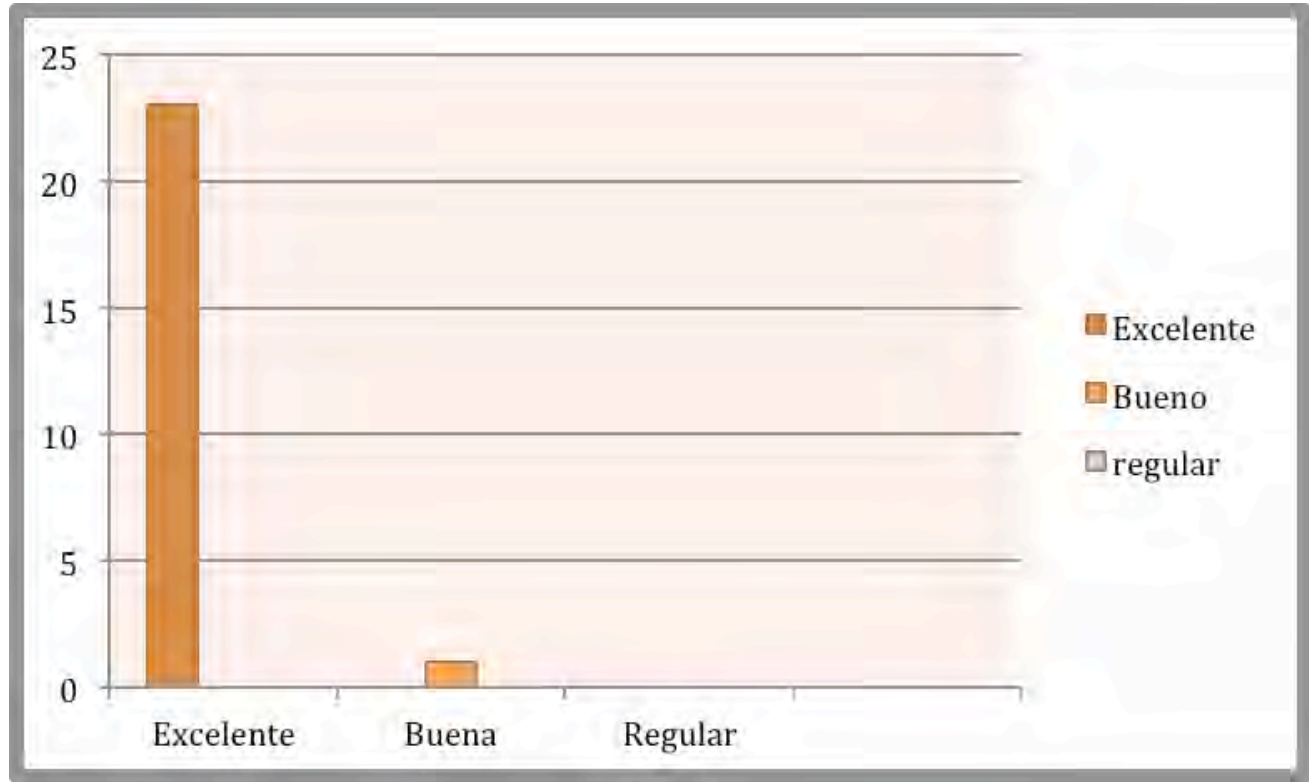

Fuente: Autor, 2015.

23 estudiantes de 24 involucrados en el proceso respondieron que les pareció Excelente, mostrándose satisfechos y emocionados por las actividades y estrategias implementadas dentro del mismo, agradecieron a los coordinadores por su participación, y a su directora de grupo por permitir el desarrollo de MAAE.

Tabla 11: Con qué valor te identificaste en el transcurso de las actividades formativas del proyecto, puede seleccionar varias opciones

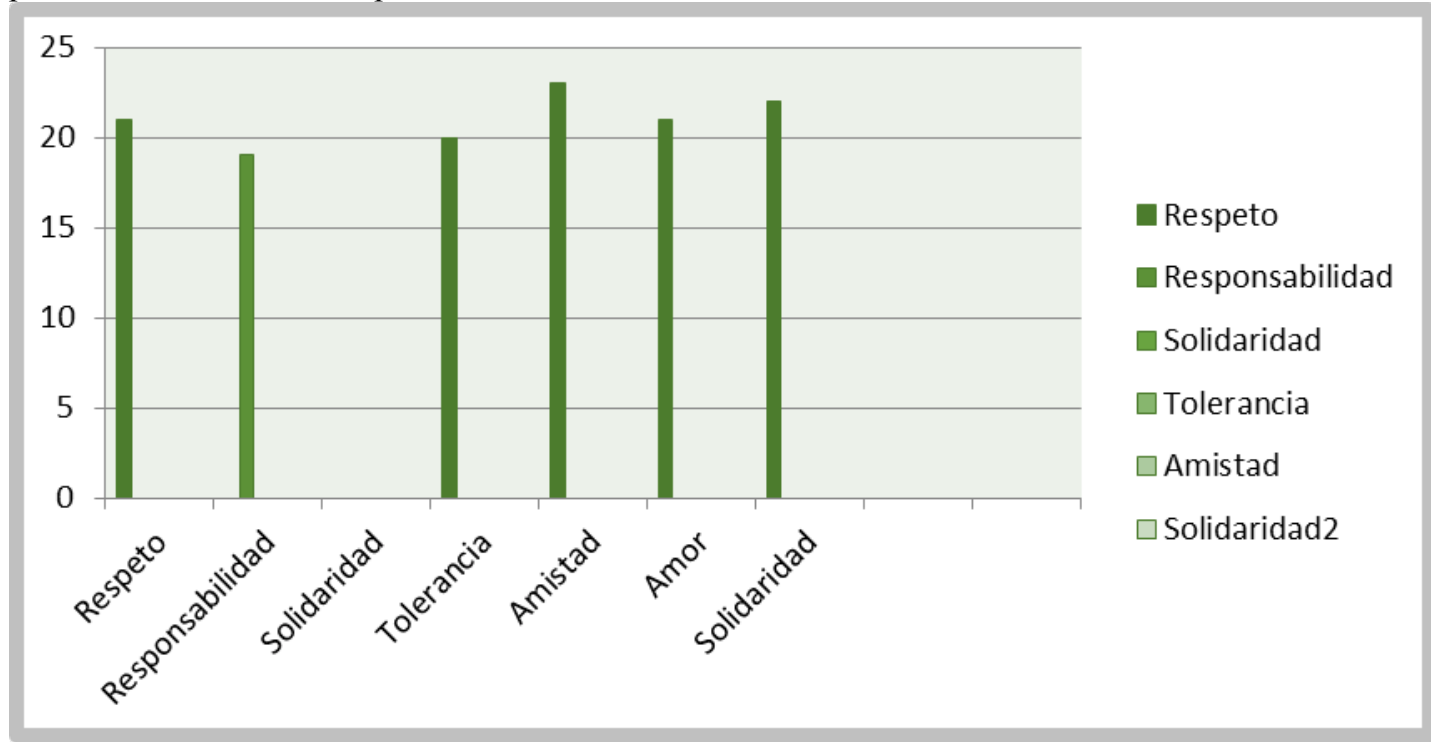

Fuente: Autor, 2015. 
Se puede concluir que la mayoría de los estudiantes escogieron todas las opciones puesto que las estrategias involucraban el fomento y desarrollo de los valores principales que todo ser humano debe dominar y exhibir, por lo tanto es satisfactorio ya que se evidencia que los objetivos definidos fueron cumplidos a cabalidad.

Tabla 12: ¿Consideras necesario que se sigan desarrollando proyectos de este tipo?

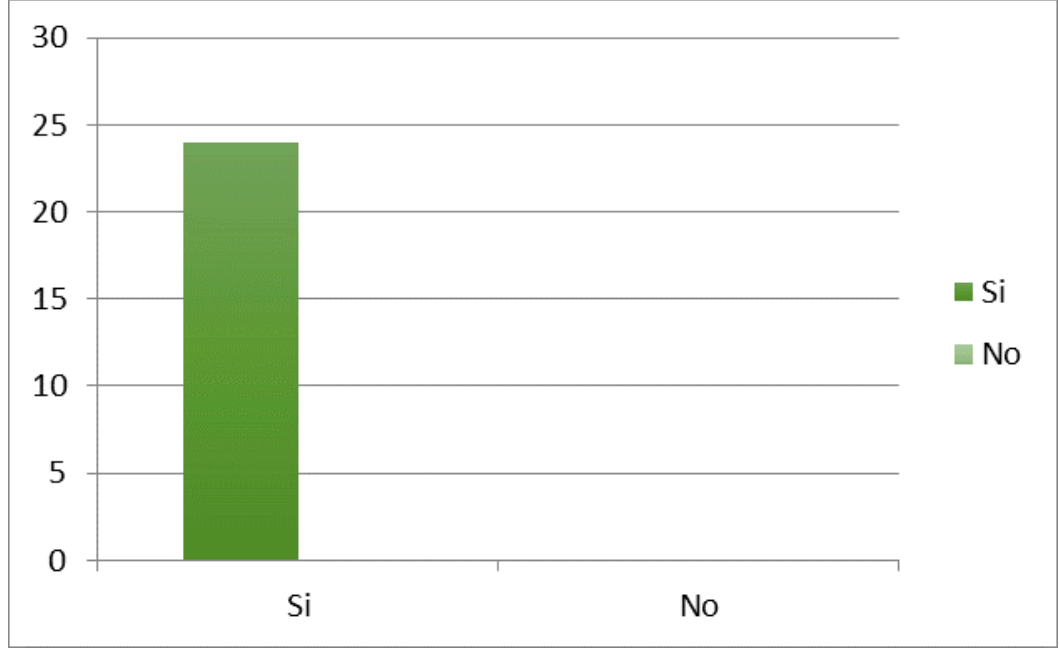

Fuente: Autor, 2015.

Tabla 13: ¿Consideras que el proyecto fortaleció los lazos afectivos con tus compañeros, familiares y docentes?

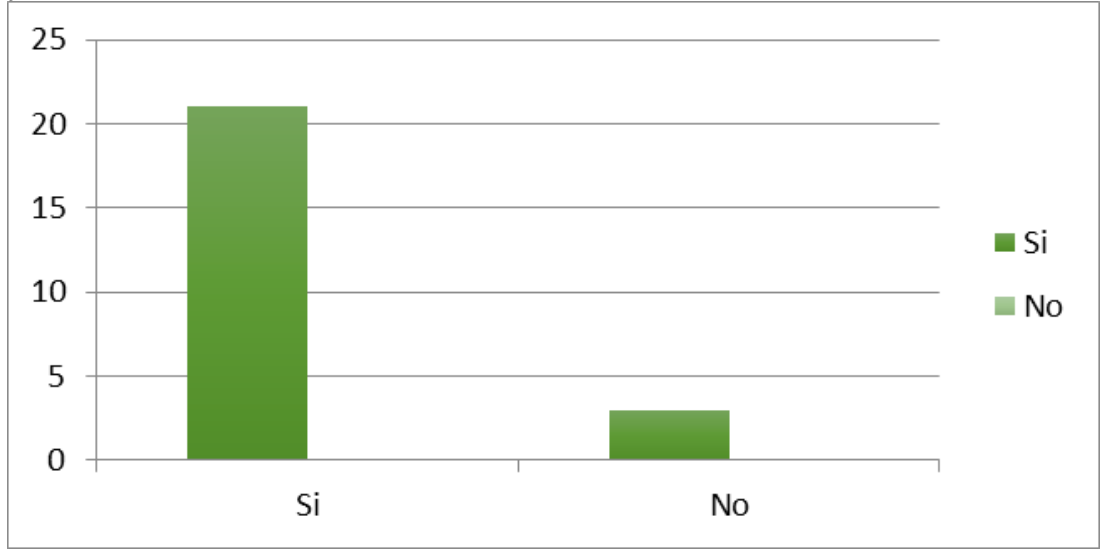

Fuente: Autor, 2015.

Se pudo apreciar que la participación de los estudiantes en el desarrollo de las estrategias de MAAE, potencializó la vinculación de los medios audiovisuales en el proceso de aprendizaje. Esto les permitió a la población implicada conocer algunas de sus fortalezas y debilidades, mejorar en los lazos afectivos dentro y fuera de la comunidad estudiantil así como los lazos familiares, por ende hicieron uso de uso de sus valores para mejorar los procesos comunicativos y el desempeño académico mejoro notablemente, todo debido a que las actividades ofrecidas fueron dinámicas ya que 
buscaban incentivar la fomento de valores humanos, quedaron en su totalidad satisfechos y desean en su totalidad que se sigan realizando este tipo de proyectos transversales formativos.

Tabla 14: ¿Qué recomendaciones nos das para mejorar dentro del proyecto MAAE?

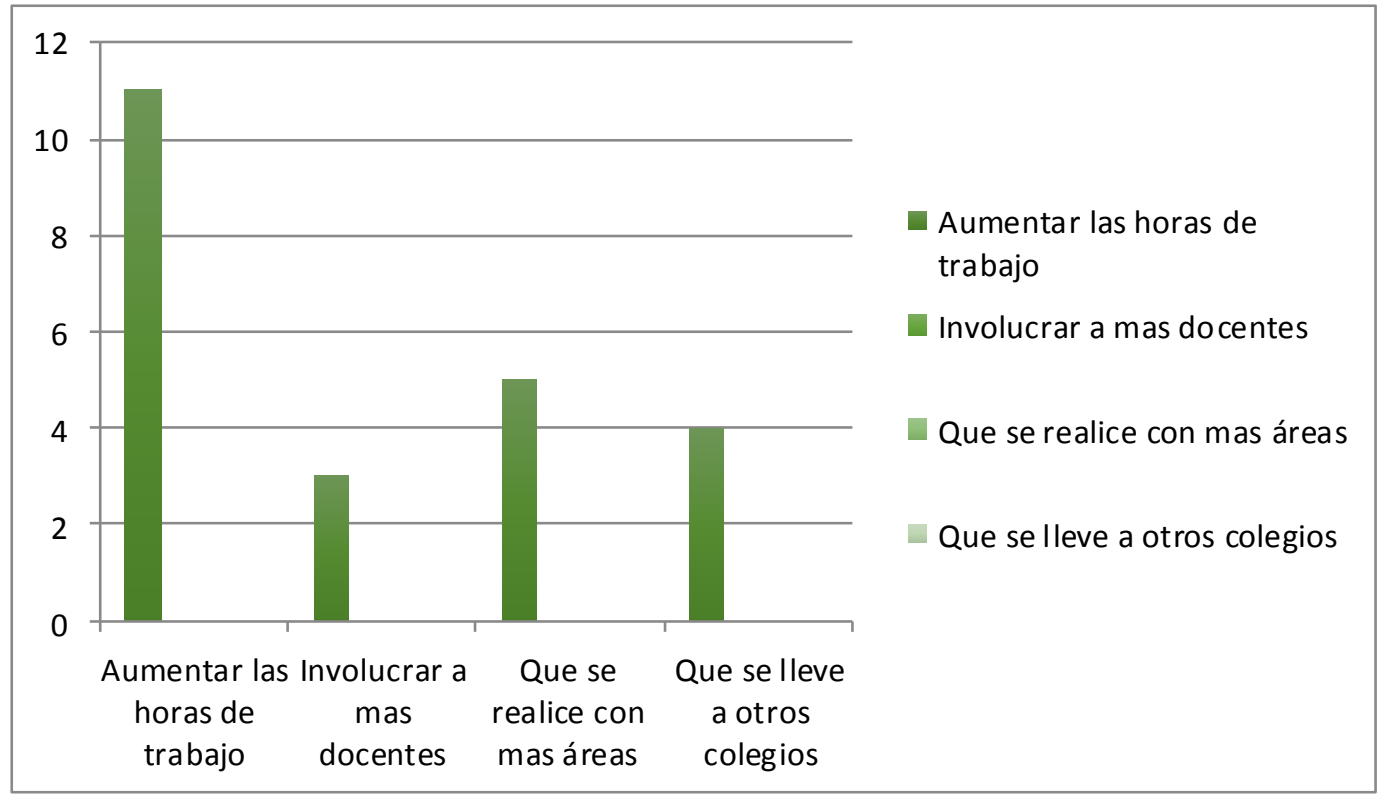

Fuente: Autor, 2015.

Si bien es cierto que se notó una mejoría en el proceso de fortalecimiento de valores y en uso y apropiación de la tecnología en el aula al finalizar las estrategias, no se debe olvidar que modificar conductas marcadas es sumamente complicado, por lo tanto es necesario por ende que las estrategias propuestas en esta investigación se implementen desde el inicio del año lectivo por los y las docentes, además que se desarrolle de manera transversal en todas las áreas y que se logre expandir este proyecto a otras instituciones para que conozcan la iniciativa y mejoren sus procesos académicos y el ambiente escolar.

Fomentar los valores desde los grados iniciales del sistema educativo debe ser prioritario, pero esta debe hacerse de forma lúdica y no impuesta pues de lo contrario el estudiante la vera como obligación y no como un mecanismo para acceder al conocimiento y enriquecer su proceso comunicativo.

Presentar a los estudiantes diferentes tipos de materiales y recursos educativos médate el uso de TIC y los medios audiovisuales hará más enriquecedora la experiencia de enseñanza.

\section{Conclusiones}

El desarrollo de la investigación logro promover el desarrollo social a través del uso de los medios audiovisuales y las TIC para el procesamiento de la información de manera adecuada en los estudiantes y establecimiento de procesos comunicativos para la obtención y la apropiación de conocimientos que favorecen la prevención de algunos conflictos.

A través de la investigación se determinó que existe en los estudiantes agrado por la observación de videos que los llevaron a la reflexión.

De acuerdo con lo manifestado por las Docentes directoras de grados cuarto y corroborado por la Psicóloga de la Institución Educativa hubo incidencia positiva en el cambio de comportamiento en la mayoría de los estudiantes investigados luego de aplicada la estrategia, por tal razón se mejoró la convivencia al interior del salón. 
En una reunión con los padres de familia se corroboro los planteamientos de la psicóloga, los cuales manifestaron que sus hijos durante el proceso y después se mostraron activos-participativos y una mejora notable en cuanto a vocabularios inapropiados y antivalores.

El prototipo web quedo como repositorio de recursos educativos mediados por los medios audiovisuales para posteriores aplicación del proyecto en diferentes contextos y con diversos propósitos.

Otro problema que disminuyó fueron los índices de bullyng o matoneo escolar en la institución, puesto que debido al fortalecimiento de los valores y el conocimiento de historias y experiencias personales se notó una postura de tolerancia y respeto por parte de la población.

El Semillero de investigación MAAE se implementó como una estrategia formativa para la realización de proyectos de investigación dentro de la institución, este grupo conformado por docentes, alumnos y padres de familia se comprometió a seguir resolviendo problemáticas que los atañen con el fin de fortalecer la cultura investigativa en el departamento y a su vez armonizar el contexto para que el proceso de enseñanza-aprendizaje sea eficaz.

\section{Recomendaciones}

Se recomienda aplicar esta estrategia en otros grados dado el nivel de aceptación, motivación y resultados obtenidos en esta investigación.

Ampliar el número de sesiones de trabajo colaborativo y ejecución de propuestas de innovación y pedagogía audiovisual.

Uso frecuente de la plataforma MAAE como una plataforma innovadora para la realización de actividades apropiadas que además de fortalecer valores estimula al desarrollo de destrezas básicas del pensamiento.

Involucrar más activamente a Padres de Familia en estas actividades ya que son ellos los pilares principales en la formación en valores desde el núcleo familiar.

Transversal izar el proyecto a todas las áreas, puesto que se evidencia la eficacia que tiene el uso pedagógico ce los medios audiovisuales en la educación 


\section{REFERENCIAS}

AIKEN. (2014). Programa de inclusión social "Cine Arte (Cine Para Todos)". Montería, Colombia.

Basanta, E. M. (2001). La formación ética en las instituciones de Educación superior. Recuperado de: http://www.campus-oei.org/revista/deloslectores/173Basanta.PDF

Bustos, R. (2009). Propuesta de estrategias en diversos planteles para orientar a alumnos motivarlos en su vocación. Montería, Córdoba, Colombia.

Briceño. (2000). Estudio de campo de carácter descriptivo, para orientar la labor docente. Docente en Colombia, Universidad de Santander.

Carretero, G. (2012). Proyecto "Educación en valores y Medios Audiovisuales en el IES Villa de Vícar".

Cardona, A. (2000). Formación de valores: Teoría, reflexiones y propuestas. México D. F., México: Grijalbo.

Cortina, A. (2000). El mundo de los valores: Ética mínima y educación. Bogotá, Colombia: El Búho.

Marqués, P. (2000). Impacto de las TIC en educación: funciones y limitaciones. Barcelona, España: UAB.

Frisancho, S. (2001). ¿Educación en valores o formación moral?: Algo más que sólo una discusión acerca de términos. Lima, Perú: PUCP-CISE.

OEI. (2002): Proyecto educación y cultura para la paz. En: OEI. Boletín 24. http://www.campusoei.org/valores/pecpperu.htm

Frondizi, R. (1968). ¿Qué son los valores? México D. F., México: Fondo de Cultura Económica.

García, S. (2001). Dirección por valores (DpV) y liderazgo postconvencional. Recuperado de: www.managementbyvalues.com/docs/dpv.pdf

Garza, T. J. y Patiño, G. S. (2000). Educación en Valores. México D. F., México: Editorial Trillas.

Gómez, J. R. (2004). Las TIC en la educación. Blog personal "Principios valores y virtudes" Recuperado de: http://portal.educ.ar/debates/educacionytic/formacion-docente/principiosvalores-y-virtudes.php

College of Computing. (1997). GVU's 8th WWW user survey. Atlanta, Estados Unidos: Georgia Institute of Technology.

Palencia, A. (2013). Proyectos de transversalización de TIC en el aula, MEDUSA. Córdoba, Colombia: Ministerio de Educación Colombiana (Colegio Patio Bonito).

Palencia, A. (Noviembre de 2014). MEDUSA: Modelo para la gestión de tecnologías en la educación. Congreso Iberoamericano de Ciencia, Tecnología, Innovación y Educación, celebrado en Buenos Aires, Argentina.

Santana, B. (1997). Introducing the Technophobia/Technophilia Debate: Some Comments on the Information Age. Los Ángeles, Estados Unidos: UCLA.

Juan Pablo II. (2002). Un nuevo foro para la proclamación del Evangelio. Mensaje del Santo Padre Juan Pablo II, para la Jornada Mundial de las Comunicaciones Sociales. Recuperado de: http://www.multimedios.org/docs/d001091/

Guevara, B., Zambrano, A. y Evies, A. (2007). ¿Para qué educar en valores? Educación en valores, $1(7), 76-86$.

UNESCO. (2010). Teaching and Learning for a Sustainable Future: The Practice of Citizenship. Associated Schools Project. París, Francia: UNESCO.

\section{SOBRE EL AUTOR}

José Antonio Correa Padilla: Licenciado en Informática y Medios Audiovisuales en la Universidad de Córdoba, Coordinador del grupo de investigación CYMTED-L (Mediaciones tecnológicas y Lenguaje para el Desarrollo Humano) Monitor de investigación para metodología de la investigación Cualitativa. 